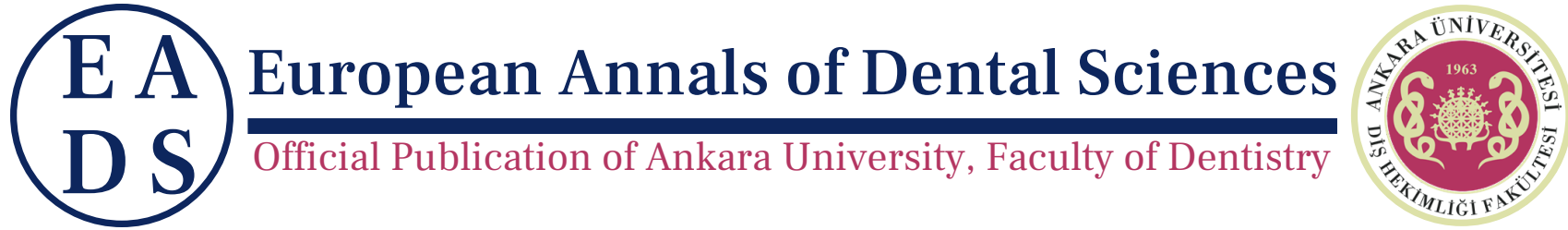

EADS, 2021, 48 (1), 23-27

\title{
A Rare Presentation of Radicular Cyst: A Case Report and Review of Literature
}

\author{
Rohan Jagtap $\odot$ 1,*, Nick Shuff $\odot$ 2, Maram Bawazir $\odot 3$, Michelle Briner \\ Garrido $\odot$, Indraneel Bhattacharyya $\odot 3$ and Matthew Hansen $\odot 2$
}

\author{
${ }^{1}$ University of Mississippi Medical Center School of Dentistry, Division of Oral and Maxillofacial Radiology \\ Department of Care Planning and Restorative Sciences, Jackson, MS, USA and ${ }^{2}$ BeamReaders, Inc. \\ Kennewick, WA, USA and ${ }^{3}$ University of Florida College of Dentistry, Department of Oral and Maxillofacial \\ Diagnostic Sciences, Gainesville, FL, USA and ${ }^{4}$ University of Missouri-Kansas City School of Dentistry \\ Department of Oral Pathology, Radiology, and Medicine, Kansas City, MO, USA \\ *Corresponding Author; drrohanjagtap@gmail.com
}

\begin{abstract}
The radicular cyst is the most common inflammatory odontogenic cyst in the jaws. It is a periapical lesion associated with non-vital teeth in the tooth-bearing regions of the jaws with a slight male predilection. A radicular cyst is typically asymptomatic, but if large or secondarily infected may cause swelling. The usual radiographic appearance of a radicular cyst is that of a periapical radiolucent lesion. This case report documents a rare case of 61-year-old male with a mixed-density periapical lesion diagnosed as a radicular cyst. The lesion presented as a well-defined, expansile, space occupying, corticated, sclerotic, hydraulic, unilocular, mixed density lesion, associated with the right mandibular second premolar that was predominantly radiolucent with scattered foci of radiopacities. Microscopic examination revealed fragments of lining epithelium along with small fragments of inflamed fibrous connective tissue, aggregates of necrotic cellular debris, and bacterial colonies intermixed with foci of dystrophic calcifications. The histopathological diagnosis was a radicular cyst with dystrophic calcification. Although rare, this entity should be considered in the differential diagnosis of mixed-density periapical lesions. Complete history and proper diagnosis is important in this type of rare cases as treatment varies between a radicular cyst and other odontogenic neoplasms.
\end{abstract}

Key words: dystrophic calcification; mixed periapical lesion; radicular cyst; residual cyst

\section{Introduction}

Approximately $60 \%$ of the radicular cysts are found in the maxilla with a propensity for the anterior maxillary teeth. Radicular cyst has a slight male predilection and is most commonly found during the third to sixth decades. ${ }^{1,2}$ It arises from chronic apical periodontitis, itself the product of root canal infection and pulpal necrosis. The inflammatory cells found in chronic apical periodontitis release cytokines and growth factors which induces epithelial rests of Malassez proliferation. The proliferation of epithelial rests of Malassez leads to formation of a radicular cyst. ${ }^{3}$

Radicular cyst pathogenesis is a complex process that has been proposed to involve aspects of the osmotic pressure theory, abscess theory, and complex epithelial-stromal interactions. ${ }^{4-7}$ The exact prevalence of radicular cysts is debated, most likely due to a large number of these lesions not undergoing routine biopsy. ${ }^{8}$ The classical radiographic presentation of radicular cysts is that of a radiolucent entity with a welldefined, expansile, corticated borders that appears hydraulic in nature; however, there have been cases described in the literature where internal calcifications are present causing confusion leading to a different subset of differential diagnoses and treatment strategies. ${ }^{7}$

Radiographically apparent internal calcifications within radicular cysts have rarely been reported in the literature. ${ }^{8}$ This case presents an unusual radiographic appearance of a radicular cyst and demonstrates the importance of clinical examination along with radiographic features to obtain a more accurate differential diagnosis and appropriate treatment plan. In this report we present a diagnostically challenging case of a radicular cyst with internal calcifications. 


\section{Case Report}

A 61-year-old male with a history of facial swelling one week in duration reported to his local hospital emergency department. The physician prescribed penicillin and recommended a dental referral. His dentist made a panoramic radiograph and, upon visualization of three periapical lesions, referred him to the Oral and Maxillofacial Surgery Department at the University of Florida College of Dentistry (UFCOD) for further evaluation. The clinician performed a cone-beam CT scan and referred study to the Department of Oral and Maxillofacial Radiology for radiographic interpretation of all three lesions.

The panoramic illustrates three periapical lesions: two in the maxilla and one in the mandible. Two lesions in maxilla are well-localized, partially corticated, entirely radiolucent entities and one in the mandible is well-defined, corticated, mixed-density entity that appears hydraulic in nature in the right parasymphyseal region of the mandible (Figure 1a). The CBCT scan demonstrated a well-defined, expansile, thinned, partly corticated, hydraulic in appearance, unilocular periapical radiolucent entity associated with teeth $5 \& 6$, and 10 . There is no evidence of calcification in the internal content of lesion, and the density of lesion is similar to the soft tissue density. There is thinning and possible disruption of the buccal and palatal cortical plates. The inferior border of right maxillary sinus appears to be displaced superiorly. There is possible external root resorption of 6 . There is extrusion of the obturation material apical to 10 with possible external root resorption (Figure $1 \mathrm{~b}$ ). The radiographic appearance is suggestive of radicular cysts.

Upon CBCT review of the mandibular lesion, the lesion was noted to be a well-defined, expansile, space occupying, corticated, sclerotic, hydraulic, unilocular, mixed density lesion in the right mandible extending from mesial of 29 to the missing 32 anterior-posteriorly and from alveolar crest to the inferior border of mandible superior-inferiorly. The right IAN canal appears to be displaced inferiorly. There is evidence of calcification in the internal content of lesion. There is buccolingual cortex expansion with possible disruption/attenuation of the lingual cortex. 29 is involved and radiolucency encompasses an apical third of 29. Three is external root resorption of the apical area of 29 (Figure 1c and 1d). The radiographic features of the lesion are not pathognomonic to a particular entity, though the appearance of the lesion is suggestive of calcifying odontogenic cyst vs. calcifying epithelial odontogenic tumor. Calcifying odontogenic cyst and calcifying epithelial odontogenic tumor were considered most likely in the differential. Histopathological examination was performed on all three lesions for definitive diagnosis. Microscopic examination of the incisional biopsy specimen revealed fragments of lining epithelium along with small fragments of inflamed fibrous connective tissue, aggregates of necrotic cellular debris, and bacterial colonies intermixed with foci of dystrophic calcifications (Figure 2A). The lining epithelium consisted of 3-4 layers of non-keratinized stratified squamous epithelial cells displaying changes secondary to inflammation (Figure 2B). The lining was attached to inflamed connective tissue in some areas while in other areas was found to be floating within the specimen. Large aggregates of necrotic cellular debris and cholesterol clefts with multinucleated giant cells were noted in the specimen. Intermixed within these necrotic zones were foci of dystrophic calcification exhibiting a basophilic appearance and a variable mineralization pattern (Figure $2 \mathrm{C}$ ). Smaller aggregates of red blood cells and areas of hemosiderin pigmentation were noted. The fibrous connective tissue which exhibits a scattered inflammatory cell infiltrate consisting of aggregates of neutrophils, macrophages, and lymphocytes. In addition, small aggregates of dystrophic calcifications are also seen in this framework (Figure 2D).

The histological investigation determined all three lesions to be radicular cysts with the radiopaque entities seen in the mandibular lesion identified as cholesterol granulomas and multiple foci of calcification.

\section{Discussion}

Radicular cyst is a type of inflammatory odontogenic cyst, of endodontic origin, and is associated with non-vital teeth. Radicular cysts with dystrophic calcifications in microscopic examinations have been reported in the literature; however, it rarely has been demonstrated as well-defined calcifications in radiographic evaluation. ${ }^{3}$ A retrospective study done by Lin et al. ${ }^{1}$ in Taiwan reported dystrophic calcification in $16.2 \%$ of the periapical cysts. The most common location for the radicular cyst is an anterior maxilla mainly associated with incisors and canine but, in our case, we had a radicular cyst with internal calcification foci found in the mandible near the apex of the right second premolar. Male to female ratio in the radicular cyst is $1.6: 1$, which was consistent in our case report being reported in a male patient. ${ }^{9,10}$ The usual radiographic appearance of a radicular cyst is documented as a radiolucent, welldefined cortical border with a size of a lesion more than $2 \mathrm{~cm}$ in diameter. ${ }^{2}$ This rare case of the radicular cyst with calcification has a size of $2.5 \mathrm{~cm} \times 3 \mathrm{~cm}$.

Ramos-Perezl et al. ${ }^{3}$ have also reported a radicular cyst with extensive radiopaque calcification in the maxilla that was related to the periapical area of a heavily restored tooth with substandard endodontic treatment. A $2 \mathrm{~cm}$ hyperdense foci with enlargement and disruption of the buccal cortical plate was noticed. Our case had two small radiopaque entities in the well-defined radiolucent lesion of $0.5 \mathrm{~cm}$ diameter each. In the Ramos-Perezl case, due to the extensive mixed lesion that showed buccal cortical bone distraction, a differential diagnosis of benign fibro-osseous lesion has been considered. The histopathological analysis showed a cystic cavity lined by non-keratinized squamous epithelium, and granular calcifications were distributed throughout the specimen with evidence of cholesterol granuloma formation and chronic inflammatory changes. 3 Krithika et al. reported a case of a mixed-density lesion in the periapical region of an edentulous site during the routine radiographic assessment. ${ }^{8}$ Microscopic evaluation was consistent with a residual radicular cyst that shows dystrophic calcification, which is similar to our case. There is controversy regarding the formation of dystrophic calcification within inflammatory odontogenic cysts. The formation of such calcifications within a radicular cyst are thought to originate from a pulse granuloma. Giant-cell hyaline angiopathy, which is synonym for a pulse granuloma, is believed to be a degenerative vascular process or a foreign body reaction. These foci of pulse granuloma represent an eosinophilic material with a corrugated periphery of condensed collagen surrounded by inflammatory cells like lymphocytes and multinucleated giant cells. Moreover, they are commonly seen in the cystic wall of inflammatory odontogenic cysts. ${ }^{11}$ Pulse granuloma represents an inflammatory exudate pool which ultimately undergo fibrosis and occasionally forms dystrophic calcification. ${ }^{12}$ Some authors had proposed the theory of calcification concomitant to actinomyces colonization in the periapical area, actinomyces being able to reach the apical tooth region through an infected root canal. A study has been done to identify actinomycotic colonies in the periapical biopsies has reported its presence in $1.8 \%$ of the cases ${ }^{13}$ - only one of those cases represented as a radiolucent lesion with radiopaque calcification resembling condensing osteitis. Many authors have reported actinomyces associated lesions in the jaw, including dentigerous cyst and os- 

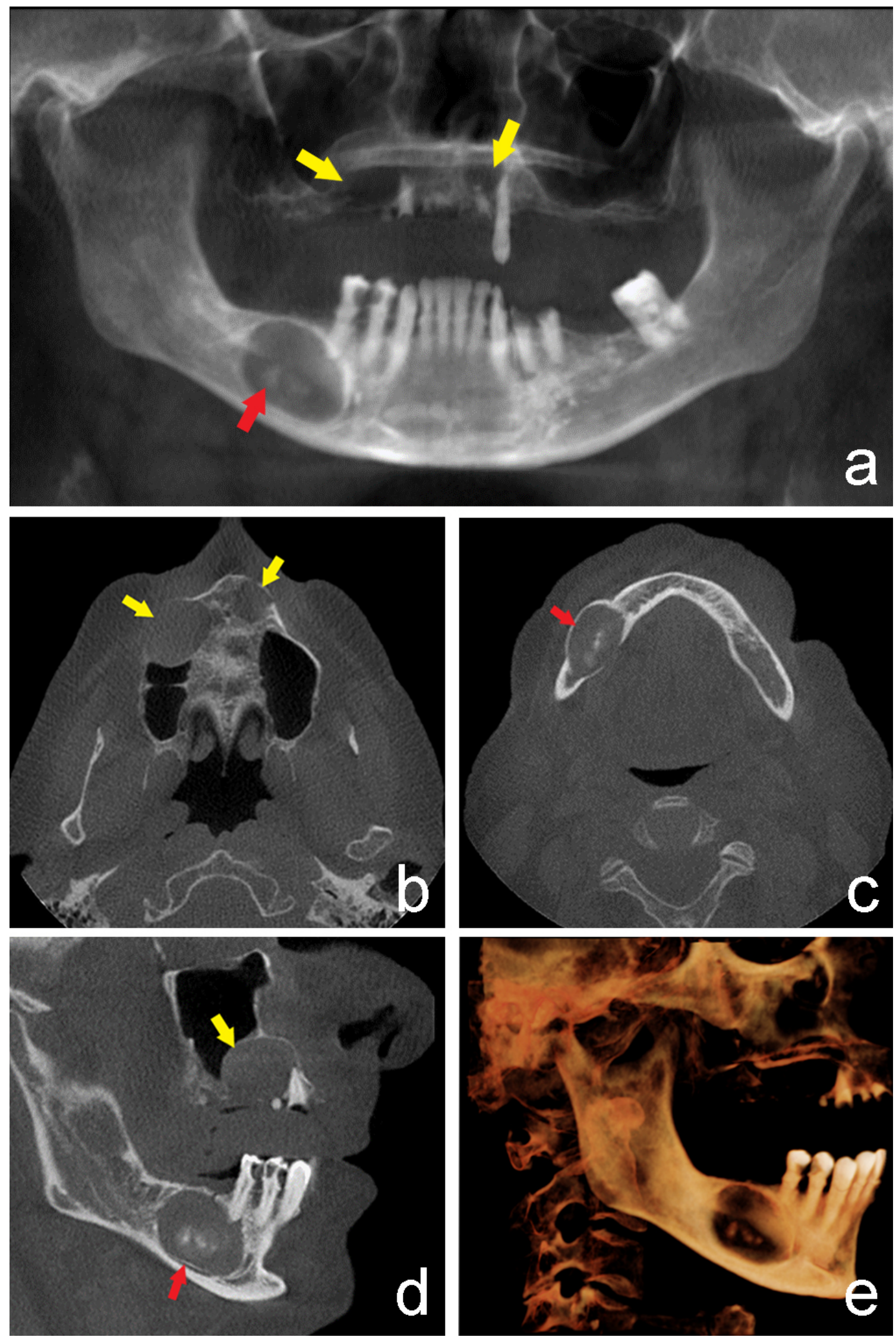

Figure 1. a: A panoramic radiograph exhibits two well-localized, partially corticated, entirely radiolucent entities in the maxilla (yellow arrows) and mixeddensity entity in the right parasymphyseal region of the mandible (red arrow). b: Axial view depict well-defined, expansile, thinned, partly corticated, hydraulic in appearance, unilocular radiolucent entities in the maxilla and thinning \& displacement of buccal cortical plate (yellow arrows). c: Axial views shows a welldefined, expansile, space occupying, corticated, sclerotic, hydraulic, unilocular, mixed density lesion in the right mandible with evidence of calcification in the internal content of lesion (red arrow). d: Parasagittal CBCT view exhibit a radiolucent entity in right maxilla with displacement of the inferior border of right maxillary sinus superiorly (yellow arrow) and a mixed-density lesion in right mandible with the right IAN canal appears to be displaced inferiorly (red arrow). e: 3D Reconstruction 

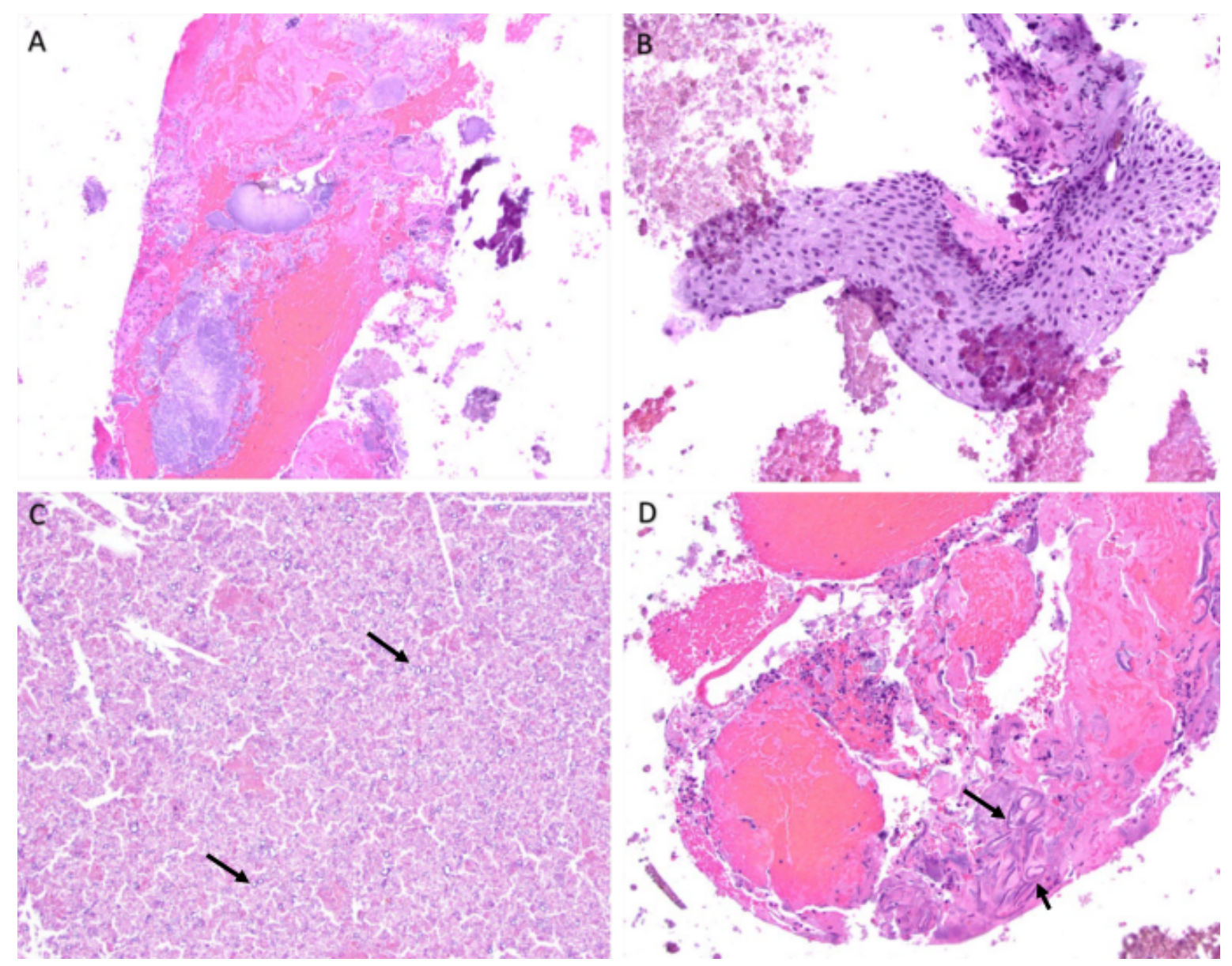

Figure 2. A: Non-specific bacterial colonies inter-mixed with inflammatory infiltrate, and aggregates of necrotic debris (10X). B: Cystic cavity lined by a nonkeratinized, hyperplasic stratified squamous epithelium (10X). C: Higher power showing foci of dystrophic calcification with a basophilic appearance in variable mineralization pattern (See arrows) (20X). D: An area showing numerous curvilinear dystrophic calcification (see arrows) and aggregates of red blood cells (20X).

teomyelitis, although few have been reported in the radicular cyst. $^{13-17}$ The dystrophic calcifications within radicular cysts are acellular and according to Bernardi, are thought to be due to the necrosis and $\mathrm{pH}$ changes that result in precipitations of serum calcium. ${ }^{5}$

The differential diagnosis of calcifying odontogenic cyst ( $\mathrm{COC} / \mathrm{CCOT}$ ) was believed to be most likely due to its variable presentation. Calcifying epithelial odontogenic tumor (CEOT) was not ideal due to the more advanced age of the patient but could not be ruled out. Similarly, the differential diagnosis of an ameloblastic fibro-odontoma was unlikely due to it typically being seen in a much younger age group. ${ }^{2,18}$ Depending on the amount of mineralized tissue within the lesion, the osseous dysplasia with simple bone cyst can be considered in a differential diagnosis as they occur in three forms: from predominantly radiolucent to radiopaque to mixed, but they rarely cause cortical bone enlargement. ${ }^{3}$ A radicular cyst was not initially considered in the list of differential diagnoses due to the radiographic evidence of internal radiopaque entities in predominantly radiolucent lesion and lack of clinical information including pulp vitality testing.

\section{Conclusion}

Internal calcifications have been described in histopathologic evaluation of radicular cysts but rarely in the radiographic evaluation. Although uncommon and infrequently documented in the literature, radicular cysts with internal calcifications are seen radiographically. With the aid of complete clinical infor- mation including pulp vitality testing, a radicular cyst may be considered for a periapical lesion with internal calcifications. The proper diagnosis of radicular cyst may lead to a more conservative treatment approach such as endodontic therapy vs enucleation, greatly improving the patient's comfort and prognosis.

This case reinforces the importance of clinical examination to aid radiographic interpretation. Oral and Maxillofacial Radiologists and Surgeons often receive little or no clinical history that is relevant in providing an accurate and focused differential diagnosis for the patient. Such information should be sought in questionable cases if it is not provided by the practitioner.

\section{Author Contributions}

RJ, NS and MB treated the patient; RJ, IB and MH collected and analyzed the data; RJ and NS led the writing.

\section{Conflict of Interest}

Author Rohan Jagtap, Author Nick Shuff, Author Maram Bawazir, Author Michelle Briner Garrido, Author Indraneel Bhattacharyya, Author Matthew Hansen declare that they have no conflict of interest. 


\section{Authors' ORCID(s)}

R.J. $\quad 0000-0002-9115-7235$

N.S. $0000-0002-6247-5090$

M.B. 0000-0002-9326-1351

M.B.G.0000-0002-0737-9848

I.B. 0000-0002-0334-9051

M.H. $0000-0001-6096-4264$

\section{References}

1. Lin HP, Chen HM, Yu CH, Kuo RC, Kuo YS, Wang YP. Clinicopathological study of 252 jaw bone periapical lesions from a private pathology laboratory. J Formos Med Assoc. 2010;109(11):810-8. doi:10.1016/s0929-6646(10)60126-x.

2. White SC, Pharoah MJ. Oral radiology-E-Book: Principles and interpretation. Elsevier Health Sciences; 2014.

3. Ramos-Perez FM, Pontual Ados A, França TR, Pontual ML, Beltrão RV, Perez DE. Mixed periapical lesion: an atypical radicular cyst with extensive calcifications. Braz Dent J. 2014;25(5):447-50. doi:10.1590/0103-6440201300235.

4. Bando Y, Henderson B, Meghji S, Poole S, Harris M. Immunocytochemical localization of inflammatory cytokines and vascular adhesion receptors in radicular cysts. J Oral Pathol Med. 1993;22(5):221-7. doi:10.1111/j.16000714.1993.tb01060.x.

5. Bernardi L, Visioli F, Nör C, Rados PV. Radicular Cyst: An Update of the Biological Factors Related to Lining Epithelium. J Endod. 2015;41(12):1951-61. doi:10.1016/j.joen.2015.08.036.

6. Nair PN. Apical periodontitis: a dynamic encounter between root canal infection and host response. $\mathrm{Pe}$ riodontol 2000. 1997;13:121-48. doi:10.1111/j.16000757.1997.tbooo98.x.

7. Nair PN. New perspectives on radicular cysts: do they heal? Int Endod J. 1998;31(3):155-60. doi:10.1046/j.13652591.1998.00146.x.

8. Krithika C, Kota S, Gopal KS, Koteeswaran D. Mixed periapical lesion: differential diagnosis of a case. Dentomaxillofac Radiol. 2011;40(3):191-4. doi:10.1259/dmfr/89370676.

9. Shear M, Speight P. Radicular Cyst and Residual Cyst. In: Cysts of the Oral and Maxillofacial Regions. Blackwell Munksgaard; 2007. p. 123-142. doi:10.1002/9780470759769.ch11.

10. Koju S, Chaurasia N, Marla V, Niroula D, Poudel P. Radicular cyst of the anterior maxilla: An insight into the most common inflammatory cyst of the jaws. J Dent Res Rev. 2019;6(1):26-29. doi:10.4103/jdrr.jdrr_64_18.

11. Kotrashetti VS, Angadi PV, Mane DR, Hallikerimath SR. Oral pulse granuloma associated with keratocystic odontogenic tumor: Report of a case and review on etiopathogenesis. Ann Maxillofac Surg. 2011;1(1):83-6. doi:10.4103/22310746.83153 .

12. Neville BW, Damm DD, Allen CM, Chi AC. Oral and maxillofacial pathology. Elsevier Health Sciences; 2015.

13. Hirshberg A, Tsesis I, Metzger Z, Kaplan I. Periapical actinomycosis: a clinicopathologic study. Oral Surg Oral Med Oral Pathol Oral Radiol Endod. 2003;95(5):614-20. doi:10.1067/moe.2003.87.

14. Fergus HS, Savord EG. Actinomycosis involving a periapical cyst in the anterior maxilla. Report of a case. Oral Surg Oral Med Oral Pathol. 1980;49(5):390-3. doi:10.1016/00304220(80)90280-7.

15. Martinelli C, Rulli MA. Periapical cyst associated with actinomycosis. Oral Surg Oral Med Oral Pathol. 1967;24(6):817-20. doi:10.1016/0030-4220(67)90519-1.

16. Shibasaki M, Iwai $\mathrm{T}$, Chikumaru H, Mitsudo K, Inayama Y, Tohnai I. Actinomyces-associated calcifications in a dentigerous cyst of the mandible. J Craniofac Surg. 2013;24(3):e311-4. doi:10.1097/SCS.ob013e31828f2e72.

17. Tek M, Metin M, Sener I, Bereket C, Tokac M, Kazancioglu $\mathrm{HO}$, et al. The predominant bacteria isolated from radicular cysts. Head Face Med. 2013;9:25. doi:10.1186/1746-160x9-25.

18. Reddy R, Jagtap R, Kashtwari D, Hambrook CC, FreburgHoffmeister DL, Islam MN, et al. Benign fibro-osseous lesion and odontoma of the mandible: A report of a rare, hybrid lesion and review of literature. J Oral Maxillofac Surg Med Pathol. 2019;31(6):415-419. doi:10.1016/j.ajoms.2019.05.002. 